\title{
Transport equations with resting phases
}

\author{
T. HILLEN \\ Department of Mathematical and Statistical Sciences, University of Alberta, \\ Edmonton T6G 2G1, Canada \\ (email: thillen@ualberta.ca)
}

(Received 21 August 2002; revised 12 May 2003)

\begin{abstract}
We study a transport model for populations whose individuals move according to a velocity jump process and stop moving in areas which provide shelter or food. This model has direct applications in ecology (e.g. homeranges, territoriality, stream ecosystems, travelling waves) or cellular biology (e.g. movement of bacteria or movement of proteins in the cell nucleus). In this paper we consider a general model from a mathematical point of view. This provides general insight into the features of these models, which in turn is useful in the modelling process. We consider a singular perturbation expansion and show that the leading order term of the outer solution satisfies a reaction-advection-diffusion equation. The advective term describes taxis toward homeranges or toward regions of shelter. The reaction terms are given by "effective" birth and death rates. Within this framework, the parameters of the reactionadvection-diffusion model (like mobility, drift, birth or death rates) are directly related to the individual movement behaviour of the species at hand (like velocity, frequency of directional changes, response to spatial in-homogeneities, death, or reproduction). We prove that in a homogeneous environment the diffusion limit approximates the solution of the resting-phase transport model to second order in the perturbation parameter.
\end{abstract}

\section{Introduction}

A widely used class of mathematical models to describe spatial spread of growing populations are reaction-diffusion models (e.g. see Murray [18] or Britton [4]). They are based on an uncorrelated random walk (Brownian motion) and they assume instantaneous birth, neglecting resting periods due to birth events.

The assumption of uncorrelated random walk has been extended to models which are based on correlated random walk and which lead to transport equations (e.g. Stroock [23], Alt [1], Othmer et al. [19], Hadeler [11], Dickinson [10], Hillen \& Othmer [13, 20]). The parameters for transport models are mean speed, mean turning rates and turning-angle distribution: parameters which can be measured from the movement paths of individuals. For that reason the transport models are more closely related to the natural process than the diffusion-based models. In fact, using appropriate scaling analysis, the transport model leads to reaction-diffusion models. In particular, in Hillen \& Othmer [13], a theory has been introduced to obtain the parabolic limit of a transport model in a general and transparent way.

In this paper, we extend the modelling with transport equations to include periods of resting where the individuals do not move. The reasons are manifold and range from shelter, den sites, or nests, to hunting spots, or feeding grounds. We give examples below. 
Since the decision when to move and when to rest is an individual one, it is natural to study this dynamic in the framework of transport models (rather than diffusion models). In Othmer \& Hillen [20] a resting-phase transport model with constant stopping rates was already formulated. In this paper we extend that model to include spatially variable stopping rates (inhomogeneous environment) and we give proofs for the various results (which are not contained in Othmer \& Hillen [20]).

The model presented here combines a transport model for correlated random walk with resting periods on preferred locations. Using a singular perturbation analysis it is shown that the long time asymptotics (i.e. outer solution) are described by a reactionadvection-diffusion model. It appears that the diffusion coefficient is proportional to the mean fraction of the population which is moving and the birth term is proportional to the mean fraction of resting particles. The spatial non-homogeneities lead to a drift term toward preferred areas.

Typically, reproductions and deaths occur on a slower time scale than the random walk. The ratio of these two corresponding time scales defines a small parameter $(\varepsilon)$. A singular perturbation analysis leads to reaction-advection-diffusion equations (parabolic limit). We show that the inner solution relaxes to a homogeneous distribution and that the outer expansion is well described by a reaction-advection-diffusion model with effective birth and death rates. We match inner and outer expansions and we prove that, in the spatial homogeneous case with linear birth and death rates, the long time asymptotics are approximated by the outer expansion to second order in the perturbation parameter. We discuss various special cases, which include small perturbations to homogeneous initial conditions, the case of large or small fraction of resting particles and we compare the results to a resting-phase diffusion model.

\subsection{Applications}

Models for movement including resting-phases are relevant in ecology and also in cellular biology:

Recent studies of stream ecosystems use reaction-diffusion models to investigate the drift paradox [17, 21]. The drift paradox occurs for aquatic insects, like the Baetidis mayflies and stoneflies, who occur as persistent populations in streams. The larvae are relatively immobile on the bottom of the stream (resting), while the adults are able to swim and as they rise from the bottom they are transported downstream. Although individuals are drained from that location, the population as a whole remains persistent (drift paradox). The inclusion of moving-resting dynamics, as in Pachepsky et al. [21] sheds a new light on possible resolutions of the drift paradox.

The dynamics of oriented movement (searching) and resting is also important to understand the survival of endangered species in fragmented habitats. In Carroll \& Lamberson [6] the territoriality of spotted owls was studied. In their model the juvenile owls are assumed to search for suitable territories (moving), while the adults have already occupied a suitable territory (resting). Carroll and Lamberson study a compartmental model of habitat clusters, which does not include any spatial information. To include the given landscape in a model, a transport model, like the one studied here, would be a natural starting point. 
Another application of a similar nature occurs for modelling the formation of homeranges. Homeranges provide shelter and include feeding grounds. In many cases they include a den site for resting and reproduction. Homeranges for wolves have been studied by Briscoe et al. [3] without inclusion of resting phases. There the formation of homeranges was triggered by scent marking. It would be interesting to see the influence of resting-moving dynamics on the home range formation.

Lewis \& Schmitz [16] and Hadeler \& Lewis [12] study the effect of resting-moving dynamics on the invasion of microbes. Certain microbes reproduce while they are stationary and only when they are not reproducing they can be spread spatially by wind or by water. In both papers a resting-phase Fisher model is investigated, which divides the whole population into a moving part, which does not reproduce, and a resting part, which only can reproduce. The model of Lewis et al. is a special case of the model studied here.

In collaboration with J. Müller, we are currently investigating homeranges of bats. The movement of individual bats shows interesting interactions of moving and resting dynamics. In detailed studies the movement path's of ten individual bats has been recorded. From this data set we plan to estimate mean speed, turning rates, turn-angle distributions and spatially dependent stopping rates.

In collaboration with M. Lewis and J. M. Lee, we are currently working on an model for prey-taxis. The predator stops movement at locations with high prey density. This process in turn leads to prey-taxis in the parabolic limit [14].

A totally different application occurs for the movement of proteins in the cell nucleus. In fluorescence-recovery-after-photobleaching experiments it was shown that certain proteins in the cell nucleus show movement patterns which cannot be described by simple diffusion. In Carrero et al. [5] a resting-phase diffusion model was used to successfully analyze the fluorescence recovery curves. In addition, with use of this model it is possible to predict the average number of bound (=resting) proteins. This information cannot be directly determined in experiments.

Cosner \& Lou [8] and Belgacem \& Cosner [2] consider a reaction-diffusion model with drift toward better environments. They study the benefit for populations which use this strategy. In fact, their model is a special case of the parabolic limit equation derived here (3.14). In Cosner et al., the modelling of the corresponding drift term is done ad hoc. The asymptotic scaling analysis as done here relates the drift mechanism to individual movement behaviour and it gives an a posteriori justification of the model used by Cosner et al.

\subsection{The model}

To model a population whose members are moving according to a correlated random walk and which rest occasionally, we split the total population density $N(t, x)$ into a density $p(t, x, v)$ of individuals moving with velocity $v \in V$ and a density $r(t, x)$ for particles resting at $x \in \Omega$. The velocity set $V$ is assumed to be bounded with $\omega=|V|$ and symmetric of the form that $v \in V$ implies $-v \in V$. We study a model for $(p, r)$ which is based on the following assumptions:

(1) The pure movement process is a velocity jump process

$$
p_{t}+v \cdot \nabla p=-\mu p+\mu \int_{V} T\left(v, v^{\prime}\right) p\left(., ., v^{\prime}\right) d v^{\prime} .
$$


We assume that the kernel $T\left(v, v^{\prime}\right)$ satisfies the following basic assumptions (T1)(T4), which were first introduced in Hillen \& Othmer [13]:

(T1) $T\left(v, v^{\prime}\right) \geqslant 0, \quad \int_{V} T\left(v, v^{\prime}\right) d v=1, \quad$ and $\quad \int_{V} \int_{V} T^{2}\left(v, v^{\prime}\right) d v^{\prime} d v<\infty$.

(T2) There exist some $u_{0} \geqslant 0$ with $u_{0} \neq 0$, some integer $N$ and a constant $\rho>0$ such that for all $\left(v, v^{\prime}\right) \in V \times V$

$$
u_{0}(v) \leqslant T^{N}\left(v^{\prime}, v\right) \leqslant \rho u_{0}(v)
$$

where the $N$ th iterate of $T$ is

$$
T^{N}\left(v, v^{\prime}\right):=\int \ldots \int T\left(v, w_{1}\right) T\left(w_{1}, w_{2}\right) \cdots T\left(w_{N-1}, v^{\prime}\right) d w_{1} \ldots d w_{N-1} .
$$

(T3) We introduce an integral operator $\mathscr{T}$ by

$$
\mathscr{T} p=\int_{V} T\left(v, v^{\prime}\right) p\left(v^{\prime}\right) d v
$$

and we assume that $\|\mathscr{T}\|_{\langle 1\rangle^{\perp}}<1$, where $\langle 1\rangle^{\perp}$ denotes the orthogonal complement of the subspace $\langle 1\rangle \subset L^{2}(V)$ of functions constant in $v$.

(T4) $\int_{V} T\left(v, v^{\prime}\right) d v^{\prime}=1$.

(2) There is a spatially dependent rate $\alpha(x)>0$ such that individuals stop moving at location $x$ with that rate $\alpha(x)$.

(3) At rest particles give birth at a rate $g(N) \geqslant 0$ ( $g$ for 'gain').

(4) Individuals start moving with constant rate $\beta>0$ and they choose a direction with equal distribution on $V$.

(5) Death occurs for moving and resting particles at the same rate $l(N) \geqslant 0$. (l for 'loss').

We denote the turning operator as $\mathscr{L}:=-\mu(I-\mathscr{T})$ and Proposition 1.1 of Hillen \& Othmer [13] applies:

Proposition 1.1 Assume (T1)-(T4). Then

(1) 0 is a simple eigenvalue of $\mathscr{L}$ with eigenfunction $\phi(v) \equiv 1$.

(2) There exists an orthogonal decomposition $L^{2}(V)=\langle 1\rangle \oplus\langle 1\rangle^{\perp}$, and for all $\psi \in\langle 1\rangle^{\perp}$ we have

$$
\int \psi \mathscr{L} \psi d v \leqslant-v_{2}\|\psi\|_{L^{2}(V)}^{2}, \quad \text { with } \quad v_{2} \equiv \mu\left(1-\|\mathscr{T}\|_{\langle 1\rangle^{\perp}}\right) .
$$

(3) Each eigenvalue $\lambda \neq 0$ satisfies $-2 \mu<\operatorname{Re} \lambda \leqslant-v_{2}<0$, and there is no other positive eigenfunction.

(4) $\|\mathscr{L}\|_{\mathscr{L}\left(L^{2}(V), L^{2}(V)\right)} \leqslant 2 \mu$. 
(5) $\mathscr{L}$ restricted to $\langle 1\rangle^{\perp} \subset L^{2}(V)$ has a linear inverse $\mathscr{F}$ with norm

$$
\|\mathscr{F}\|_{\mathscr{L}\left(\langle 1\rangle^{\perp},\langle 1\rangle^{\perp}\right)} \leqslant \frac{1}{v_{2}} .
$$

The resting-phase transport model reads

$$
\begin{aligned}
p_{t}+v \cdot \nabla p & =\mathscr{L} p-\alpha(x) p+\frac{\beta}{\omega} r-l(N) p \\
r_{t} & =\alpha(x) \int_{V} p(., ., v) d v-\beta r+g(N) r-l(N) r,
\end{aligned}
$$

where the total particle density $N$ is given by

$$
N(t, x)=\int_{V} p(t, x, v) d v+r(t, x) .
$$

We assume compactly supported initial data on $\Omega=\mathbb{R}^{n}$, which are $L^{2}$-integrable on $\mathbb{R}^{n} \times V$. Then the solution will have compact support as long as it exists. Note that the velocity $v$ appears as a parameter in (1.2). Hence, no conditions on $\partial V$ are required.

For later use we define the pure kinetic birth-death process without any movement by

$$
\dot{u}=f(u):=g(u) u-l(u) u .
$$

We study the parabolic limit of (1.2), (1.3) in the framework of singular perturbation theory and matching asymptotic expansions. For that purpose we identify appropriate scalings of space and time (parabolic scaling). As pointed out in Hillen \& Othmer [13], these scalings occur quite naturally for biological populations. For a given small quantity $\varepsilon>0$ we consider fast and slow time scales, $t$ and $\tau=\varepsilon^{2} t$, respectively, on microscopic and macroscopic space scales $x$ and $\xi=\varepsilon x$, respectively. In singular perturbation theory one splits the dynamics into two parts. The first part (inner solution) describes the relaxation of the initial data on the fast time scale $t$. The outer solution describes the long time behaviour on the slow time scale $\tau$. The initial data for the outer solution are not directly specified by the initial data of the original problem (1.2), (1.3). This can be achieved by matching inner and outer expansions in an intermediate region. Roughly speaking, the asymptotics of the inner solution for $t \rightarrow \infty$ defines the initial condition for the outer solution at $\tau=0$.

We begin with local and global existence of solutions to (1.2), (1.3). Then we study the outer solution $(\tilde{p}, \tilde{r})$ which leads to the diffusion limit equation (3.14). This gives approximations of second order in the perturbation parameter $\varepsilon$ (Theorem 5.5). After that, we study the inner solution and we illustrate that both parts match correctly.

\section{Assumptions, existence, positivity}

Here we collect a set of basic assumptions which we use throughout the paper.

(A1) $f \in C^{1}(\mathbb{R}), g, l \in C_{b}^{1}(\mathbb{R})$ and $l\left(N^{*}\right) \geqslant g\left(N^{*}\right)$ for some $N^{*}>0$.

(A2) The initial data

$$
p(0, x, v)=\varphi(x, v) \geqslant 0, \quad r(0, x)=\psi(x) \geqslant 0
$$


are spatially compact supported with $\int \varphi(x, v) d v+\psi(x) \leqslant N^{*}, \varphi(x,.) \in L^{2}(V)$ for all $x \in \mathbb{R}^{n}$ and $\varphi(., v) \in C^{0, \sigma}\left(\mathbb{R}^{n}\right)$ for almost all $v \in V$ and some $0<\sigma<1$.

(A3) $\alpha(x)>0$, and $\alpha \in H^{1}\left(\mathbb{R}^{n}\right) \cap W^{1, \infty}\left(\mathbb{R}^{n}\right)$.

(A4) $V=s S^{n-1}$ or $V=B_{s}(0)$ for some $s>0$, and $\omega=|V|$.

(A5) $T>0$ is fixed.

The assumption (A5) is not important for our existence result. But the assumption will be used for the approximation estimates later. Hence we choose to state it here together with the other assumptions.

First we show global existence:

Theorem 2.1 Assume (T1)-(T4) and (A1)-(A4). For each pair of initial data $\varphi, \psi$ with $\varphi \in D(\Phi)$ and $\psi \in L^{2}\left(\mathbb{R}^{n}\right)$ with $\varphi(., v), \psi \in L^{1}\left(\mathbb{R}^{n}\right)$ there is a unique solution $(p, r)$ of $(1.2)$, (1.3) with

$$
p \in C^{1}\left([0, \infty), L^{2}\left(\mathbb{R}^{n} \times V\right)\right) \cap C^{0}([0, \infty), D(\Phi)), \quad r \in C^{1}\left([0, \infty), L^{2}\left(\mathbb{R}^{n}\right)\right)
$$

and $p(0,)=.\varphi$ and $r(0)=\psi$, where $\Phi$ is the shift operator as defined below.

Proof We collect a number of arguments and apply standart semigroup theory:

(1) The shift operator $\Phi=-v \cdot \nabla$ with domain

$$
D(\Phi)=\left\{\varphi \in L^{2}\left(\mathbb{R}^{n} \times V\right): \varphi(., v) \in H^{1}\left(\mathbb{R}^{n}\right)\right\}
$$

generates a strongly continuous, positive, unitary group of translations on $L^{2}\left(\mathbb{R}^{n} \times\right.$ $V$ ) (see also Dautray \& Lions [9, Ch XXI, Sect. 2, Prop 1]).

(2) The nonlinearities are of the form $g(\varphi) \psi, l(\varphi) \psi$ with $\varphi, \psi \in L^{2}\left(\mathbb{R}^{n} \times V\right)$ with bounded and differentiable rates $g$ and $l$. Hence $g(\varphi) \psi, l(\varphi) \psi \in L^{2}\left(\mathbb{R}^{n} \times V\right)$. Moreover, the mappings $((\varphi, \psi) \mapsto g(\varphi) \psi)$ and $((\varphi, \psi) \mapsto l(\varphi) \psi)$ are globally Lipschitz continuous on both $L^{2}\left(\mathbb{R}^{n} \times V\right)^{2}$ and $H^{1}\left(\mathbb{R}^{n} \times V\right)^{2}$.

(3) With $\alpha$ given as in (A3) also the mappings $p \mapsto \alpha(x) p$, and $p \mapsto \alpha(x) \int p d v$ are Lipschitz continuous on both spaces $L^{2}\left(\mathbb{R}^{n} \times V\right)^{2}$ and $H^{1}\left(\mathbb{R}^{n} \times V\right)^{2}$.

(4) The turning operator $\mathscr{L}$ is linear and compact.

Hence local and global existence of solutions to $(1.2,1.3)$ follows from standard Perroniteration and perturbation arguments (e.g. see Taylor [24] or Pazy [22]).

System (1.2), (1.3) also preserves positivity:

Lemma 2.2 Under the assumptions of the previous Theorem we have

$$
p(t, x, v) \geqslant 0 \quad \text { and } \quad r(t, x) \geqslant 0 .
$$

Proof If we assume that the solution $(p(t), r(t))$ becomes negative somewhere then there is a first time $t_{0}$ where either $p\left(t_{0}\right)=0$, or $r\left(t_{0}\right)=0$, or both. 
In the first case, $p\left(t_{0}\right)=0$, we find for $t=t_{0}$ from (1.2) that

$$
p_{t}+v \cdot \nabla p=\frac{\beta}{\omega} r \geqslant 0
$$

In the second case, $r\left(t_{0}\right)=0$, we find from (1.3) that at $t=t_{0}$

$$
r_{t}=\alpha(x) \int_{V} p(., ., v) d v \geqslant 0 .
$$

Note that $\Phi=-v \cdot \nabla$ generates a positive group of translations. Hence in both cases $p(t)$, or $r(t)$ cannot decay below 0 .

\section{The outer expansion}

\subsection{Scaling}

In Hillen \& Othmer [13] we introduced the scaling of

$$
\tau=\varepsilon^{2} t, \quad \xi=\varepsilon x,
$$

for small $0<\varepsilon \leqslant 1$.

We assume that there is a large number of individual directional changes per birth or death event. This means that birth and death events occur on a much slower time scale than the random walk process. As $t$ denotes the fast time scale (as for the random walk) and $\tau$ denotes the slow time scale we assume that the pure kinetics is described by

$$
u_{\tau}=\tilde{f}(u)
$$

or equivalently

$$
u_{t}=\varepsilon^{2} \tilde{f}(u)
$$

We identify as follows:

$$
f(u)=\varepsilon^{2} \tilde{f}(u)=\varepsilon^{2}(\tilde{g}(u) u-\tilde{l}(u) u) .
$$

System (1.2), (1.3) in the new variables $(\tau, \xi)$ reads

$$
\begin{aligned}
\varepsilon^{2} \tilde{p}_{\tau}+\varepsilon v \cdot \nabla_{\xi} \tilde{p} & =\mathscr{L} \tilde{p}-\tilde{\alpha} \tilde{p}+\frac{\beta}{\omega} \tilde{r}-\varepsilon^{2} \tilde{l}(\tilde{N}) \tilde{p} \\
\varepsilon^{2} \tilde{r}_{\tau} & =\tilde{\alpha} \int \tilde{p}(., ., v) d v-\beta \tilde{r}+\varepsilon^{2} \tilde{g}(\tilde{N}) \tilde{r}-\varepsilon^{2} \tilde{l}(\tilde{N}) \tilde{r}
\end{aligned}
$$

where $\tilde{p}(\tau, \xi, v)=p\left(\tau / \varepsilon^{2}, \xi / \varepsilon, v\right), \tilde{r}(\tau, \xi)=r\left(\tau / \varepsilon^{2}, \xi / \varepsilon\right)$, and $\tilde{\alpha}(\xi)=\alpha(\xi / \varepsilon)$. We consider series expansions in $\varepsilon$ up to order $k>2$ :

$$
\begin{aligned}
\tilde{p}(\tau, \xi, v):=\sum_{j=0}^{k} \varepsilon^{j} p_{j}(\tau, \xi, v), & \tilde{r}(\tau, \xi):=\sum_{j=0}^{k} \varepsilon^{j} r_{j}(\tau, \xi) \\
\tilde{N}(\tau, \xi):=\sum_{j=0}^{k} \varepsilon^{j} N_{j}(\tau, \xi), & N_{j}(\tau, \xi)=r_{j}(\tau, \xi)+\int_{V} p_{j}(\tau, \xi, v) d v, \quad 0 \leqslant j \leqslant k .
\end{aligned}
$$


We expand the nonlinearities $\tilde{g}, \tilde{l}$ according to this representation:

$$
\begin{aligned}
& \tilde{g}(\tilde{N})=\tilde{g}\left(N_{0}\right)+\tilde{g}^{\prime}\left(N_{0}\right)\left(\sum_{j=1}^{k} \varepsilon^{j} N_{j}\right)+\text { l.o.t. } \\
& \tilde{l}(\tilde{N})=\tilde{l}\left(N_{0}\right)+\tilde{l}^{\prime}\left(N_{0}\right)\left(\sum_{j=1}^{k} \varepsilon^{j} N_{j}\right)+\text { l.o.t.. }
\end{aligned}
$$

\subsection{Formal derivation of the diffusion limit}

We introduce all of the above expansions into system (3.1), (3.2) and collect orders of $\varepsilon$. During this section we neglect the subscript $\xi$ at the $\nabla$-operator:

$$
\begin{array}{ll}
\varepsilon^{0}: & \left\{\begin{array}{l}
0=\mathscr{L} p_{0}-\tilde{\alpha} p_{0}+\frac{\beta}{\omega} r_{0} \\
0=\tilde{\alpha} \int p_{0} d v-\beta r_{0}
\end{array}\right. \\
\varepsilon^{1}: & \left\{\begin{array}{r}
v \cdot \nabla p_{0}=\mathscr{L} p_{1}-\tilde{\alpha} p_{1}+\frac{\beta}{\omega} r_{1} \\
0=\tilde{\alpha} \int p_{1} d v-\beta r_{1}
\end{array}\right. \\
\varepsilon^{2}:\left\{\begin{array}{r}
p_{0, \tau}+v \cdot \nabla p_{1}=\mathscr{L} p_{2}-\tilde{\alpha} p_{2}+\frac{\beta}{\omega} r_{2}-\tilde{l}\left(N_{0}\right) p_{0} \\
r_{0, \tau}=\tilde{\alpha} \int p_{2} d v-\beta r_{2}+\tilde{g}\left(N_{0}\right) r_{0}-\tilde{l}\left(N_{0}\right) r_{0} .
\end{array}\right.
\end{array}
$$

From (3.3) it follows that

$$
r_{0}=\frac{\tilde{\alpha}}{\beta} \int p_{0} d v
$$

Hence the first equation of (3.3) reads

$$
0=\mathscr{L} p_{0}-\tilde{\alpha} p_{0}+\frac{\tilde{\alpha}}{\omega} \int p_{0} d v=: \mathscr{L}_{\alpha} p_{0}
$$

where the operator $\mathscr{L}_{\alpha}(\xi)$ is given for $\tilde{\alpha}(\xi)>0$ and $\psi \in L^{2}(V)$ by

$$
\begin{aligned}
\mathscr{L}_{\alpha}(\xi)[\psi(v)]= & -(\mu+\tilde{\alpha}(\xi)) \psi(v) \\
& +(\mu+\tilde{\alpha}(\xi)) \int_{V}\left(\frac{\mu}{\mu+\tilde{\alpha}(\xi)} T\left(v, v^{\prime}\right)+\frac{\tilde{\alpha}(\xi)}{(\mu+\tilde{\alpha}(\xi)) \omega}\right) \psi\left(v^{\prime}\right) d v^{\prime}
\end{aligned}
$$

We denote the modified turning kernel by

$$
T_{\alpha}\left(\xi, v, v^{\prime}\right):=\frac{\mu}{\mu+\tilde{\alpha}(\xi)} T\left(v, v^{\prime}\right)+\frac{\tilde{\alpha}(\xi)}{(\mu+\tilde{\alpha}(\xi)) \omega}
$$

and $\mathscr{T}_{\alpha}$ is the integral operator defined by $T_{\alpha}$. For $\alpha=$ constant the formula for $L_{\alpha}$ and $T_{\alpha}$ were already given in Othmer \& Hillen [20].

Lemma 3.1 For each $\xi \in \mathbb{R}^{n}$ the kernel $T_{\alpha}$ satisfies conditions (T1)-(T4).

Proof Since $T$ is assumed to satisfy (T1)-(T4) it satisfies $T \geqslant 0$. Hence, for $\tilde{\alpha}(\xi)>0$ we have $T_{\alpha}\left(\xi, v, v^{\prime}\right)>0$ for all $\left(v, v^{\prime}\right) \in V^{2}$. Then (T2) and the first condition of (T1) follow. 
Integration of the kernel gives:

$$
\int T_{\alpha}\left(\xi, v, v^{\prime}\right) d v=\frac{\mu}{\mu+\tilde{\alpha}(\xi)}+\frac{\tilde{\alpha}(\xi)}{\mu+\tilde{\alpha}(\xi)}=1=\int T_{\alpha}\left(\xi, v, v^{\prime}\right) d v^{\prime} .
$$

Hence (T4) and the second condition in (T1) are satisfied. Moreover

$$
\iint T_{\alpha}^{2}\left(\xi, v, v^{\prime}\right) d v d v^{\prime}=\left(\mu^{2} \iint T^{2}\left(v, v^{\prime}\right) d v d v^{\prime}+2 \mu \tilde{\alpha}(\xi)+\tilde{\alpha}(\xi)^{2}\right)(\mu+\tilde{\alpha}(\xi))^{-2}<\infty .
$$

Then (T1) is satisfied as well and it remains to check (T3):

$$
\begin{aligned}
\left\|\mathscr{T}_{\alpha}\right\|_{\langle 1\rangle^{\perp}} & =\sup _{\psi \in L^{2}(V),\|\psi\|=1, \int \psi d v=0}\left|\int \frac{\mu}{\mu+\tilde{\alpha}(\xi)} T\left(v, v^{\prime}\right) \psi\left(v^{\prime}\right) d v^{\prime}+\frac{\tilde{\alpha}(\xi)}{(\mu+\tilde{\alpha}(\xi)) \omega} \int \psi(v) d v\right| \\
& \leqslant\|\mathscr{T}\|_{\langle 1\rangle^{\perp}}<1 .
\end{aligned}
$$

Hence Proposition 1.1 applies for $\mathscr{L}_{\alpha}(\xi)$, and we have the following properties.

Corollary 3.2 Assume (T1)-(T4) for $T\left(v, v^{\prime}\right)$ and let $\mathscr{L}_{\alpha}(\xi)$ be defined by (3.7) for $\tilde{\alpha}>0$. Then for each $\xi \in \mathbb{R}^{n}$

(1) 0 is a simple eigenvalue of $\mathscr{L}_{\alpha}(\xi)$ and the corresponding eigenfunction is $\phi(v) \equiv 1$.

(2) There is a decomposition $L^{2}(V)=\langle 1\rangle \oplus\langle 1\rangle^{\perp}$ and for all $\psi \in\langle 1\rangle^{\perp}$

$$
\int \psi \mathscr{L}_{\alpha}(\xi) \psi d v \leqslant-v_{\alpha}(\xi)\|\psi\|_{L^{2}(V)}^{2}, \quad \text { where } \quad v_{\alpha}(\xi) \equiv(\mu+\tilde{\alpha}(\xi))\left(1-\left\|\mathscr{T}_{\alpha}\right\|_{\langle 1\rangle^{\perp}}\right) .
$$

(3) All nonzero eigenvalues $\lambda$ satisfy $-2(\mu+\tilde{\alpha}(\xi))<R e \lambda \leqslant-v_{\alpha}(\xi)<0$, and to within scalar multiples there is no other positive eigenfunction.

(4) $\left\|\mathscr{L}_{\alpha}(\xi)\right\|_{\mathscr{L}\left(L^{2}(V), L^{2}(V)\right)} \leqslant 2(\mu+\tilde{\alpha}(\xi))$.

(5) $\mathscr{L}_{\alpha}(\xi)$ restricted to $\langle 1\rangle^{\perp} \subset L^{2}(V)$ has a linear inverse $\mathscr{F}_{\alpha}(\xi)$ with norm

$$
\left\|\mathscr{F}_{\alpha}(\xi)\right\|_{\mathscr{L}\left(\langle 1\rangle^{\perp},\langle 1\rangle^{\perp}\right)} \leqslant \frac{1}{v_{\alpha}(\xi)} .
$$

Now we go back to consider the $\varepsilon^{j}$-systems for $j=0,1,2$.

$\boldsymbol{\varepsilon}^{\mathbf{0}}:$ With the above Corollary it follows from (3.6) that $p_{0}=p_{0}(\tau, \xi)$ does not depend on velocity $v \in V$. Then

$$
r_{0}=\frac{\tilde{\alpha}}{\beta} \int p_{0} d v=\frac{\tilde{\alpha} \omega}{\beta} p_{0} .
$$

$\boldsymbol{\varepsilon}^{1}$ : From the second equation of (3.4) it follows that $r_{1}=\tilde{\alpha} / \beta \int p_{1} d v$, hence in the first equation of (3.4) the operator $\mathscr{L}_{\alpha}$ appears again:

$$
v \cdot \nabla p_{0}=\mathscr{L}_{\alpha} p_{1}
$$


This equation is solvable since

$$
\int\left(v \cdot \nabla p_{0}\right) d v=\int v d v \cdot \nabla p_{0}=0 .
$$

Then

$$
p_{1}=\mathscr{F}_{\alpha}\left(v \cdot \nabla p_{0}\right), \quad \text { with } \quad \mathscr{F}_{\alpha}:=\left(\left.\mathscr{L}_{\alpha}\right|_{\langle 1\rangle^{\perp}}\right)^{-1} .
$$

Since $\mathscr{F}_{\alpha}$ is one-to-one on $\langle 1\rangle^{\perp}$ we have $\int p_{1} d v=0$ and then $r_{1}=0$.

$\varepsilon^{2}$ : From the second equation of (3.5) we obtain

$$
\frac{\beta}{\omega} r_{2}=\frac{1}{\omega}\left(\tilde{\alpha} \int p_{2} d v+\tilde{g}_{0} r_{0}-\tilde{l}_{0} r_{0}-r_{0, \tau}\right),
$$

where for now we write $\tilde{g}_{0}:=\tilde{g}\left(N_{0}\right)$ and similarly for $\tilde{l}_{0}$. With (3.11) we get

$$
\frac{\beta}{\omega} r_{2}=\frac{\tilde{\alpha}}{\omega} \int p_{2} d v+\frac{\tilde{\alpha}}{\beta}\left(\tilde{g}_{0} p_{0}-\tilde{l}_{0} p_{0}-p_{0, \tau}\right) .
$$

We introduce this expression into the first equation of (3.5):

$$
p_{0, \tau}+v \cdot \nabla p_{1}=\mathscr{L}_{\alpha} p_{2}+\frac{\tilde{\alpha}}{\beta}\left(\tilde{g}_{0}-\tilde{l}_{0}\right) p_{0}-\frac{\tilde{\alpha}}{\beta} p_{0, \tau}-\tilde{l}_{0} p_{0} .
$$

The solvability condition with respect to $\mathscr{L}_{\alpha}$ reads, with use of (3.12),

$$
\int_{V}\left(1+\frac{\tilde{\alpha}}{\beta}\right) p_{0, \tau} d v+\nabla \cdot \int_{V} v \mathscr{F}_{\alpha} v d v \nabla p_{0}=\frac{\tilde{\alpha}}{\beta}\left(\tilde{g}_{0}-\tilde{l}_{0}\right) \int_{V} p_{0} d v-\tilde{l}_{0} \int_{V} p_{0} d v .
$$

Since $p_{0}$ does not depend upon velocity $v \in V$ this equation becomes

$$
\omega\left(1+\frac{\tilde{\alpha}}{\beta}\right) p_{0, \tau}+\nabla \cdot \int_{V} v \mathscr{F}_{\alpha} v d v \cdot \nabla p_{0}=\tilde{g}_{0} \frac{\tilde{\alpha}}{\beta} \omega p_{0}-\tilde{l}_{0}\left(1+\frac{\tilde{\alpha}}{\beta}\right) \omega p_{0} .
$$

Now $\tilde{g}_{0}=\tilde{g}\left(N_{0}\right)$ and

$$
N_{0}=r_{0}+\omega p_{0}=\left(1+\frac{\tilde{\alpha}}{\beta}\right) \omega p_{0} .
$$

Finally, we write the above equation as an equation for $N_{0}$. Note that the $\nabla p_{0}$-term produces an additional drift term, since $\tilde{\alpha}$ depends on space $\xi$. The parabolic limit equation reads

$$
N_{0, \tau}=\nabla\left(D_{\alpha, \beta}(\xi) \nabla N_{0}-D_{\alpha, \beta}(\xi) \frac{N_{0} \nabla \tilde{\alpha}(\xi)}{\beta+\tilde{\alpha}(\xi)}\right)+\frac{\tilde{\alpha}(\xi)}{\tilde{\alpha}(\xi)+\beta} \tilde{g}\left(N_{0}\right) N_{0}-\tilde{l}\left(N_{0}\right) N_{0}
$$

with diffusion tensor

$$
D_{\alpha, \beta}(\xi):=-\frac{\beta}{\omega(\tilde{\alpha}(\xi)+\beta)} \int v \mathscr{F}_{\alpha}(\xi) v d v
$$

Remark 3.3 (1) Due to the non-homogeneous environment we obtain a taxis term in direction of $-\nabla \tilde{\alpha}$. The population moves toward regions of high stopping probability, 
which reflects shelter and safe environment near the den sites or homeranges. This gives an a posteriori justification for a model studied by Cosner \& Lou [8], who directly assume taxis toward favorable environments.

(2) We obtain a birth rate reduced by a factor $\tilde{\alpha}(\xi) /(\tilde{\alpha}(\xi)+\beta)$. This factor describes the mean fraction of the population at a location $\xi$, which is at rest at any time.

(3) It turns out that, in general, we obtain non-isotropic diffusion. The diffusion tensor is scaled by a factor $\beta /(\tilde{\alpha}(\xi)+\beta)$, which describes the mean proportion of moving particles at location $\xi$. In $\S 6$ we will discuss special cases for the diffusion tensor $D_{\alpha, \beta}$.

\section{Inner expansion and matching}

For the inner expansion we consider the original fast time scale $t$ and the macroscopic space scale $\xi$. Then $\hat{p}(t, \xi, v)=p(t, x / \varepsilon, v)$ and $\hat{r}(t, \xi)=r(t, x / \varepsilon)$ satisfy the initial value problem

$$
\begin{gathered}
\hat{p}_{t}+\varepsilon v \cdot \nabla \hat{p}=\mathscr{L} \hat{p}-\tilde{\alpha} \hat{p}+\frac{\beta}{\omega} \hat{r}-\varepsilon^{2} \hat{l}(\hat{N}) \hat{p} \\
\hat{r}_{t}=\tilde{\alpha} \int \hat{p} d v-\beta \hat{r}+\varepsilon^{2} \hat{g}(\hat{N}) \hat{r}-\varepsilon^{2} \hat{l}(\hat{N}) \hat{r} \\
\hat{p}(0, \xi, v)=\varphi(\xi / \varepsilon, v) \quad \hat{r}(0, \xi)=\psi(\xi / \varepsilon),
\end{gathered}
$$

where $\hat{N}=\int \hat{p} d v+\hat{r}$ and $\hat{l}(\hat{N})=\tilde{l}\left(\tilde{N}\left(\varepsilon^{2} t, \xi\right)\right)$ and $\hat{g}(\hat{N})=\tilde{g}\left(\tilde{N}\left(\varepsilon^{2} t, \xi\right)\right)$.

Again, we study expansions in $\varepsilon$

$$
\begin{aligned}
\hat{p}(t, \xi, v):=\sum_{j=0}^{k} \varepsilon^{j} \hat{p}_{j}(t, \xi, v), & \hat{r}(t, \xi):=\sum_{j=0}^{k} \varepsilon^{j} \hat{r}_{j}(t, \xi) \\
\hat{N}(t, \xi),:=\sum_{j=0}^{k} \varepsilon^{j} \hat{N}_{j}(t, \xi), & \hat{N}_{j}(t, \xi)=\hat{r}_{j}(t, \xi)+\int \hat{p}_{j}(t, \xi, v) d v, \quad 0 \leqslant j \leqslant k,
\end{aligned}
$$

and we collect orders of $\varepsilon$ of order zero only:

$$
\varepsilon^{0}:\left\{\begin{array}{l}
\hat{p}_{0, t}=\mathscr{L} \hat{p}_{0}-\tilde{\alpha} \hat{p}_{0}+\frac{\beta}{\omega} \hat{r}_{0} \\
\hat{r}_{0, t}=\tilde{\alpha} \int \hat{p}_{0} d v-\beta \hat{r}_{0}
\end{array} .\right.
$$

We show that the functional

$$
\mathscr{E}(p, r):=\frac{\omega}{2} \int_{V} p^{2} d v+r \int_{V} p d v+\frac{r^{2}}{2}
$$

is a Lyapunov function of the integro-differential system (4.2). Note that $\mathscr{E}$ is bounded below by 0 , since the solutions are non-negative (Lemma 2.2 ).

Theorem 4.1 For solutions $\left(\hat{p}_{0}, \hat{r}_{0}\right)$ of (4.2) we have

$$
\frac{d}{d t} \mathscr{E}\left(\hat{p}_{0}, \hat{r}_{0}\right) \leqslant-\tilde{\alpha} \omega \int\left(\hat{p}_{0}-\frac{1}{\omega} \int \hat{p}_{0} d v^{\prime}\right)^{2} d v .
$$




\section{Proof}

$$
\begin{aligned}
& \frac{d}{d t} \frac{\omega}{2} \int \hat{p}_{0}^{2} d v=\omega \int \hat{p}_{0} \mathscr{L} \hat{p}_{0} d v-\omega \tilde{\alpha} \int \hat{p}_{0}^{2} d v+\beta \int \hat{p}_{0} d v \hat{r}_{0} \\
& \leqslant-\tilde{\alpha} \omega \int \hat{p}_{0}^{2} d v+\beta \hat{r}_{0} \int \hat{p}_{0} d v \\
& \frac{d}{d t}\left(\hat{r}_{0} \int \hat{p}_{0} d v\right)=\left(\tilde{\alpha} \int \hat{p}_{0} d v-\beta \hat{r}_{0}\right) \int \hat{p}_{0} d v+\hat{r}_{0}\left(\int \mathscr{L} \hat{p}_{0} d v-\tilde{\alpha} \int \hat{p}_{0} d v+\frac{\beta}{\omega} \int \hat{r}_{0} d v\right) \\
&=\tilde{\alpha}\left(\int \hat{p}_{0} d v\right)^{2}-(\tilde{\alpha}+\beta) \hat{r}_{0} \int \hat{p}_{0} d v+\beta \hat{r}_{0}^{2}, \\
& \frac{d}{d t} \frac{r_{0}^{2}}{2}=\tilde{\alpha} \hat{r}_{0} \int \hat{p}_{0} d v-\beta \hat{r}_{0}^{2} .
\end{aligned}
$$

From these inequalities it follows that

$$
\begin{aligned}
\frac{d}{d t} \mathscr{E}\left(\hat{p}_{0}, \hat{r}_{0}\right) & \leqslant-\omega \tilde{\alpha} \int \hat{p}_{0}^{2} d v+\tilde{\alpha}\left(\int \hat{p}_{0} d v\right)^{2} \\
& =-\tilde{\alpha} \omega \int\left(\hat{p}_{0}-\frac{1}{\omega} \int \hat{p}_{0} d v^{\prime}\right)^{2} d v
\end{aligned}
$$

For now we abbreviate the mean value by

$$
\phi_{0}(t, \xi):=\int \hat{p}_{0}\left(t, \xi, v^{\prime}\right) d v^{\prime}
$$

Since $\mathscr{E}$ is a Lyapunov function we have

$$
\lim _{t \rightarrow \infty}\left\|\hat{p}_{0}(t, \xi, .)-\frac{1}{\omega} \phi_{0}(t, \xi)\right\|_{2}=0 .
$$

Hence in the limit of $\tau \rightarrow \infty$ the function $\hat{p}_{0}$ does not depend on $v$. Then $\mathscr{L} \hat{p}_{0}=0$ and the asymptotic behaviour of (4.2) is determined by the system

$$
\begin{aligned}
\phi_{0, t} & =-\tilde{\alpha} \phi_{0}+\beta \hat{r}_{0} \\
\hat{r}_{0, t} & =\tilde{\alpha} \phi_{0}-\beta \hat{r}_{0} .
\end{aligned}
$$

The mass $\hat{N}(\xi)=\phi_{0}(\xi)+\hat{r}_{0}(\xi)$ is preserved for all $\xi \in \mathbb{R}^{n}$ and the steady state $(\beta \hat{N} /(\tilde{\alpha}+$ $\beta), \tilde{\alpha} \hat{N} /(\tilde{\alpha}+\beta))$ is asymptotically stable. The initial data

$$
p(0, \xi, v)=\varphi(\xi, v), \quad r(0, \xi)=\psi(\xi)
$$

determines $\hat{N}$ as

$$
\hat{N}(\xi)=\int \varphi(\xi, v) d v+\psi(\xi)
$$


Hence the limit of the inner solution $\left(\hat{p}_{0}, \hat{r}_{0}\right)$ for $t \rightarrow \infty$ is given by

$$
\begin{aligned}
& \hat{P}_{0}(\xi)=\frac{\beta}{\omega(\tilde{\alpha}(\xi)+\beta)}\left(\int \varphi\left(\xi, v^{\prime}\right) d v^{\prime}+\psi(\xi)\right) \\
& \hat{R}_{0}(\xi)=\frac{\tilde{\alpha}(\xi)}{\tilde{\alpha}(\xi)+\beta}\left(\int \varphi\left(\xi, v^{\prime}\right) d v^{\prime}+\psi(\xi)\right) .
\end{aligned}
$$

Matching: to match inner and outer solutions we use the asymptotic limit of the inner solution $(4.4,4.5)$ as initial condition for the outer solution. These are homogeneous in $v$, as was expected for the outer solution. The corresponding initial condition for the parabolic limit $N_{0}(\tau, \xi)$ is then given by

$$
N_{0}(0, \xi)=\omega \hat{P}_{0}(\xi)+\hat{R}_{0}(\xi)=\int_{V} \varphi(\xi, v) d v+\psi(\xi) .
$$

This matches exactly the initial conditions of the outer expansion which we used in (5.4).

\section{Spatially homogeneous environment}

In this section we consider the case of $\alpha(x)=\alpha=$ constant in more detail. We prove that the outer solution provides a good approximation. The approximation is of order $\varepsilon^{2}$ in intervals of the form $\theta / \varepsilon^{2}<t \leqslant T / \varepsilon^{2}$ on compact sets $\Omega \subset \mathbb{R}^{n}$.

First we show a global $L^{2}$-estimate for $p_{0}$. For that purpose we need some information on mean values of $p$. Let

$$
\begin{aligned}
& \check{p}(t, x):=\int p(t, x, v) d v \quad \bar{p}(t):=\int \check{p}(t, x) d x, \\
& \bar{r}(t):=\int r(t, x) d x, \quad \bar{N}(t):=\int N(t, x) d x=\bar{p}(t)+\bar{r}(t) .
\end{aligned}
$$

\section{Lemma 5.1}

$$
\bar{N}(t) \leqslant \bar{N}(0) e^{\|g\|_{\infty} t}
$$

Proof Integration of system $(1.2,1.3)$ with respect to space and velocity gives

$$
\begin{aligned}
& \bar{p}_{t}=-\alpha \bar{p}+\beta \bar{r}-\int l(N) \check{p} d x \\
& \bar{r}_{t}=\alpha \bar{p}-\beta \bar{r}+\int g(N) r d x-\int l(N) r d x .
\end{aligned}
$$

Note that due to $\int T\left(v, v^{\prime}\right) d v=1$ the $V$-integral of $\mathscr{L} p$ vanished. The above equations add to

$$
\bar{N}_{t}=\int g(N) r d x-\int l(N)(r+\check{p}) d x .
$$

where the last term is non-positive. Then $\bar{N}_{t} \leqslant\|g\|_{\infty} \bar{N}$ and we obtain (5.2).

Proposition 5.2 Assume (A1)-(A5). Let ( $p, r)$ denote a solution of (1.2), (1.3). Then for each $t$ with $0<t \leqslant T$ there is a constant $c_{1}=c_{1}\left(\alpha, \beta, \omega, \mu, \min \{l\},\|g\|_{\infty}, T\right)$ such that

$$
\|p(t, . . .)\|_{L^{2}\left(\mathbb{R}^{n} \times V\right)} \leqslant c_{1} \bar{N}(0) .
$$


Proof With use of (1.2) we obtain

$$
\begin{aligned}
\frac{d}{d t} \frac{1}{2} \int p^{2} d v & =\int p\left(-v \cdot \nabla p+\mathscr{L} p-\alpha p+\frac{\beta}{\omega} r-l(N) p\right) d v \\
& =-\nabla \cdot \frac{1}{2} \int v p^{2} d v+\int p \mathscr{L} p d v-\alpha \int p^{2} d v+\frac{\beta}{\omega} \int p d v r-l(N) \int p^{2} d v .
\end{aligned}
$$

In view of the inequality $\int p \mathscr{L} p d v \leqslant 0$ (see Proposition 1.1), integration of the equation above with respect to space gives

$$
\frac{d}{d t} \frac{1}{2}\|p\|_{L^{2}\left(\mathbb{R}^{n} \times V\right)}^{2} \leqslant-\alpha\|p\|_{L^{2}\left(\mathbb{R}^{n} \times V\right)}^{2}+\frac{\beta}{\omega} \bar{N}^{2}-\min \{l\}\|p\|_{L^{2}\left(\mathbb{R}^{n} \times V\right)}^{2} .
$$

Here we used Lemma 5.1 and the fact that $\int\left(\int p d v r\right) d x \leqslant \bar{N}^{2}$. With use of Gronwall's Lemma and Lemma 5.1 the assertion follows.

\subsection{Regularity properties of the limit equation}

Here we study regularity properties of the parabolic limit initial value problem in case of $\alpha=$ const.

$$
\begin{aligned}
N_{0, \tau} & =\nabla D_{\alpha, \beta} \nabla N_{0}+\frac{\alpha}{\alpha+\beta} \tilde{g}\left(N_{0}\right) N_{0}-\tilde{l}\left(N_{0}\right) N_{0} \\
N_{0}(0, \xi) & =\int \varphi(\xi, v) d v+\psi(\xi) .
\end{aligned}
$$

Lemma 5.3 The interval $\Gamma:=\left[0, N^{*}\right]$ is positively invariant for solutions of (5.4).

Proof A result of Chuey et al. [7] applies.

Proposition 5.4 For $\vartheta$ with $0<\vartheta<T$ we have

$$
\begin{aligned}
& \sup _{\vartheta \leqslant \tau \leqslant T}\left\|D^{2} N_{0}(\tau, .)\right\|_{\infty} \leqslant K_{\vartheta, T}\left\|N_{0}(0, .)\right\|_{C^{0, \sigma}}, \\
& \sup _{\vartheta \leqslant \tau \leqslant T}\left\|N_{0, \tau}(\tau, .)\right\|_{\infty} \leqslant \kappa_{\alpha, \beta} K_{\vartheta, T}\left\|N_{0}(0, .)\right\|_{C^{0, \sigma}}+c_{3} N^{*},
\end{aligned}
$$

where

$$
\begin{aligned}
K_{\vartheta, T} & =c_{2}\left(\vartheta^{\sigma / 2-1}+T^{\sigma / 2} 2^{\left[2 T K_{1}\left(N^{*}\right)\right]+1}\right) . \\
c_{3} & =\frac{\alpha}{\alpha+\beta}\|\tilde{g}\|_{C^{0}(\Gamma)}+\|\tilde{l}\|_{C^{0}(\Gamma)} \\
\kappa_{\alpha, \beta} & =\frac{\beta s^{2}}{\omega(\alpha+\beta) v_{\alpha}},
\end{aligned}
$$

$c_{2}$ is defined by (5.5) and $K_{1}\left(N^{*}\right)$ is given by (5.9).

Proof Since $A:=\nabla D_{\alpha, \beta} \nabla$ defines a strongly elliptic sectorial operator (see Hillen \& Othmer [13, Lemma 3.3]) it generates an analytic contraction semigroup on $C^{2+\sigma}\left(\mathbb{R}^{n}\right)$ ([15]). Moreover, for $\phi \in C^{0, \sigma}\left(\mathbb{R}^{n}\right)$, which does not grow faster than $e^{c|x|^{2}}$ for $|x| \rightarrow \infty$, we 
have the regularity property that

$$
\left\|D^{2}\left(e^{A \tau} \phi\right)\right\|_{\infty} \leqslant c_{2} \tau^{\sigma / 2-1}\|\phi\|_{C^{0, \sigma}}
$$

(See Ladyžhenskaja et al. [15, Chapt. IV, 11 formula (11.6)]). Note that for compactly supported initial data the solution $N_{0}(\tau, \xi)$ of (5.4) has exactly the correct growth behaviour as $|x| \rightarrow \infty$. This can be seen by representing the solution with fundamental solutions [15]. From the limit equation (5.4) we obtain

$$
N_{0}(\tau, \xi)=e^{A \tau} N_{0}(0, \xi)+\int_{0}^{\tau} e^{A(\tau-\theta)}\left(\frac{\alpha}{\alpha+\beta} \tilde{g}\left(N_{0}\right) N_{0}-\tilde{l}\left(N_{0}\right) N_{0}\right) d \theta
$$

which leads to

$$
\left\|N_{0}(\tau, .)\right\|_{C^{0, \sigma}} \leqslant\left\|N_{0}(0, .)\right\|_{C^{0, \sigma}}+\int_{0}^{\tau}\left\|\frac{\alpha}{\alpha+\beta} \tilde{g}\left(N_{0}\right) N_{0}-\tilde{l}\left(N_{0}\right) N_{0}\right\|_{C^{0, \sigma}} d \theta .
$$

The norm $\|\cdot\|_{C^{0, \sigma}}$ is defined as $\|\cdot\|_{C^{0, \sigma}}=\|\cdot\|_{\infty}+[.]_{\sigma}$, where [.] $]_{\sigma}$ denotes the usual Hölder seminorm. For now we define

$$
h(N):=\frac{\alpha}{\alpha+\beta} \tilde{g}(N)-\tilde{l}(N)
$$

and obtain

$$
\left\|h\left(N_{0}\right) N_{0}\right\|_{\infty} \leqslant\|h\|_{C^{0}(\Gamma)}\left\|N_{0}\right\|_{\infty} .
$$

To find a bound for the Hölder seminorm we consider $x \neq y \in \mathbb{R}^{n}$ and we get

$$
\begin{aligned}
\frac{h(N(x)) N(x)-h(N(y)) N(y)}{|x-y|^{\sigma}}= & \frac{h(N(x))-h(N(y))}{|N(x)-N(y)|} N(x) \frac{|N(x)-N(y)|}{|x-y|^{\sigma}} \\
& +h(N(y)) \frac{|N(x)-N(y)|}{|x-y|^{\sigma}} \\
\leqslant & \left(\|h\|_{C^{1}(\Gamma)} N^{*}+\|h\|_{C^{0}(\Gamma)}\right)\|N\|_{C^{0, \sigma}}
\end{aligned}
$$

Hence from (5.7) and (5.8) we obtain

$$
\left\|N_{0}(\tau, .)\right\|_{C^{0, \sigma}} \leqslant\left\|N_{0}(0, .)\right\|_{C^{0, \sigma}}+\tau\left(\|h\|_{C^{1}(\Gamma)} N^{*}+2\|h\|_{C^{0}(\Gamma)}\right) \sup _{0 \leqslant \theta \leqslant \tau}\left\|N_{0}(\theta, .)\right\|_{C^{0, \sigma}} .
$$

We denote

$$
K_{1}\left(N^{*}\right):=\|h\|_{C^{1}(\Gamma)} N^{*}+2\|h\|_{C^{0}(\Gamma)}
$$

and choose

Then we have

$$
\tau_{0}=\frac{1}{2 K_{1}\left(N^{*}\right)}
$$

$$
\sup _{0 \leqslant \theta \leqslant \tau_{0}}\left\|N_{0}(\theta, .)\right\|_{C^{0, \sigma}} \leqslant 2\left\|N_{0}(0, .)\right\|_{C^{0, \sigma}} .
$$

This estimate can be iterated such that for any $k \in \mathbb{N}$ we have

$$
\sup _{0 \leqslant \theta \leqslant k \tau_{0}}\left\|N_{0}(\theta, .)\right\|_{C^{0, \sigma}} \leqslant 2^{k}\left\|N_{0}(0, .)\right\|_{C^{0, \sigma}} .
$$


With the regularity property mentioned above (5.5) we obtain

$$
\begin{aligned}
\left\|D^{2} N_{0}(\tau, .)\right\|_{\infty} & \leqslant c_{2} \tau^{\sigma / 2-1}\left\|N_{0}(0, .)\right\|_{C^{0, \sigma}}+c_{2} \int_{0}^{\tau}(\tau-\theta)^{\sigma / 2-1}\left\|h\left(N_{0}\right) N_{0}\right\|_{C^{0, \sigma}} d \theta \\
& \leqslant c_{2} \tau^{\sigma / 2-1}\left\|N_{0}(0, .)\right\|_{C^{0, \sigma}}+c_{2} \tau^{\sigma / 2} K_{1}\left(N^{*}\right) \sup _{0 \leqslant \vartheta \leqslant \tau}\left\|N_{0}(\vartheta, .)\right\|_{C^{0, \sigma}}
\end{aligned}
$$

Then for $0<\vartheta \leqslant T$ it follows from (5.11) that

$$
\sup _{\vartheta \leqslant \tau \leqslant T}\left\|D^{2} N_{0}(\tau, .)\right\|_{\infty} \leqslant c_{2} \vartheta^{\sigma / 2-1}\left\|N_{0}(0, .)\right\|_{C^{0, \sigma}}+c_{2} T^{\sigma / 2} K_{1}\left(N^{*}\right) 2^{k}\left\|N_{0}(0, .)\right\|_{C^{0, \sigma}},
$$

with $k=\left[T / \tau_{0}\right]+1$. This proves (i) of the above Proposition.

To obtain (ii), we apply this estimate directly to the equation (5.4), and we use the fact that

$$
\left\|\nabla D_{\alpha, \beta} \nabla N_{0}(\tau, .)\right\|_{\infty} \leqslant \kappa_{\alpha, \beta}\left\|D^{2} N_{0}(\tau, .)\right\|_{\infty} .
$$

\subsection{Approximation property of the outer solution}

Here we show that for constant $f$ and $g$ the solution of the parabolic limit equation provides a second-order approximation to the solution of the transport model.

Theorem 5.5 Let the assumptions (A1)-(A5) be satisfied and assume $\tilde{g}=g \geqslant 0$ and $\tilde{l}=$ $l>0$ are constant with $(g-l) / l \neq \beta$. Suppose:

(1) The pair $(p(t, x, v), r(t, x))$ solves the resting-phase transport system (1.2), (1.3) for $0 \leqslant t \leqslant T$ with homogeneous initial conditions $p(0, x, v)=\varphi(x), r(0, x)=\psi(x)$.

(2) $N_{0}(\tau, \xi)$ solves the parabolic limit initial value problem (5.4) in $C^{0, \sigma}\left(\mathbb{R}^{n}\right)$.

(3)

$$
p_{0}(\tau, \xi):=\frac{\beta}{\omega(\alpha+\beta)} N_{0}(\tau, \xi), \quad r_{0}(\tau, \xi):=\frac{\alpha}{\alpha+\beta} N_{0}(\tau, \xi) .
$$

(4)

$$
p_{1}(\tau, \xi, v):=\mathscr{F}_{\alpha}\left(v \cdot \nabla p_{0}(\tau, \xi)\right), \quad r_{1}(\tau, \xi, v):=0
$$

We define two functions $P(\tau, \xi, v)$ and $R(\tau, \xi)$ by

$$
\begin{aligned}
\tilde{p}(\tau, \xi, v) & =p_{0}(\tau, \xi)+\varepsilon p_{1}(\tau, \xi, v)+\varepsilon^{2} P(\tau, \xi, v) \\
\tilde{r}(\tau, \xi) & =r_{0}(\tau, \xi)+\varepsilon^{2} R(\tau, \xi)
\end{aligned}
$$

and we assume that

$$
\int_{V} P d v=\frac{g-l}{l} R
$$

Then for each $\vartheta$ and $T$ with $0<\vartheta<T$ and each compact set $\Omega \subset \mathbb{R}^{n}$ there is a constant

$$
c_{4}=c_{4}\left(|\Omega|, T, \vartheta, \alpha, \beta, v_{\alpha}, s, N^{*}, \bar{N}(0),\left\|N_{0}(0, .)\right\|_{C^{0, \sigma}}\right)
$$


such that for all $t$ with $\vartheta / \varepsilon^{2}<t \leqslant T / \varepsilon^{2}$ we have

$$
\left\|p(t, . . .)-\left[p_{0}\left(\varepsilon^{2} t, .\right)+\varepsilon p_{1}\left(\varepsilon^{2} t, . . .\right)\right]\right\|_{L^{2}(\Omega \times V)}+\left\|r(t, .)-r_{0}\left(\varepsilon^{2} t, .\right)\right\|_{\infty} \leqslant c_{4} \varepsilon^{2} .
$$

Proof We show that $P$ and $R$ are bounded in appropriate norms, independent of $\varepsilon$.

The functions $p_{0}, p_{1}, r_{0}$ and $r_{1}$ are chosen to satisfy the $\varepsilon^{0}$ - and $\varepsilon^{1}$-systems (3.3), (3.4), respectively. It remains to study the $\varepsilon^{2}$-system for $P$ and $R$ :

$$
\begin{aligned}
p_{0, \tau}+v \cdot \nabla p_{1} & =\mathscr{L} P-\alpha P+\frac{\beta}{\omega} R-l \tilde{p} \\
r_{0, \tau} & =\alpha \int P d v-\beta R+g \tilde{r}-l \tilde{r} .
\end{aligned}
$$

We solve (5.15) for $\beta R$ and insert into (5.14) to obtain

$$
p_{0, \tau}+v \cdot \nabla p_{1}=\mathscr{L}_{\alpha} P-\frac{1}{\omega} r_{0, \tau}+\frac{g}{\omega}\left(r_{0}+\varepsilon^{2} R\right)-\frac{1}{\omega}\left(r_{0}+\varepsilon^{2} R\right)-l\left(p_{0}+\varepsilon p_{1}+\varepsilon^{2} P\right) .
$$

The solvability for $P$ leads to condition (5.12), since we already know that $p_{1} \in\langle 1\rangle^{\perp}$.

It follows from (5.15) that

$$
r_{0, \tau}=\left(\frac{g-l}{l}-\beta\right) B+g \tilde{r}-l \tilde{r} .
$$

Hence

$$
\|B(t, .)\|_{\infty} \leqslant\left(\frac{g-l}{l}-\beta\right)^{-1}\left[(g+l) \bar{N}(t)+\frac{\alpha}{\alpha+\beta}\left\|N_{0, \tau}(\tau, .)\right\|_{\infty}\right]
$$

where $\bar{N}(t)$ has been defined in (5.1). With Lemma 5.1 and with Proposition 5.4(ii) there is an $\varepsilon$-independent constant $c_{5}$ such that

$$
\|B(t, .)\|_{\infty} \leqslant c_{5}
$$

To find an estimate for $P$ we solve (5.15) for $R$ and substitute the resulting expression into (5.14) to obtain

$$
\mathscr{L}_{\alpha} P=p_{0, \tau}+v \cdot \nabla \mathscr{F}_{\alpha}\left(v \cdot \nabla p_{0}\right)-\frac{g \tilde{r}}{\omega}+l\left(\frac{\tilde{r}}{\omega}+\tilde{p}\right)+\frac{1}{\omega} r_{0, \tau},
$$

with $\mathscr{L}_{\alpha}$ from (3.7) and $\mathscr{F}_{\alpha}$ from Corollary 3.2. We study $L^{2}$-norms. For the last term we get

$$
\begin{aligned}
\left\|\frac{\tilde{r}}{\omega}+\tilde{p}\right\|_{L^{2}\left(\mathbb{R}^{n} \times V\right)}^{2} & =\int \frac{\tilde{r}}{\omega}\left(\frac{\tilde{r}}{\omega}+2 \int \tilde{p} d v\right)+\|\tilde{p}\|_{L^{2}}^{2} \\
& \leqslant \frac{2}{\omega^{2}} \bar{N}(\tau)^{2}+c_{1}^{2} \bar{N}(0)^{2} \\
& \leqslant c_{6} \bar{N}(0)^{2}
\end{aligned}
$$


where we used Lemma 5.1, Proposition 5.2 and the fact that $\tilde{r}(\tau, \xi)+\int \tilde{p}(\tau, \xi, v) d v \leqslant \bar{N}(\tau)$. The constant $c_{6}$ is given by

$$
c_{6}:=\frac{2}{\omega^{2}} e^{2 g T}+c_{1}
$$

From (5.18) it follows that for all compact sets $\Omega \in \mathbb{R}^{n}$ we have

$$
\begin{aligned}
\left\|\mathscr{L}_{\alpha} P(\tau, . .)\right\|_{L^{2}(\Omega \times V)}^{2} \leqslant & \omega|\Omega|\left\|N_{0, \tau}(\tau, .)\right\|_{\infty}^{2}+\omega\left\|\mathscr{F}_{\alpha}\right\|^{2} s^{2}|\Omega|\left\|D^{2} p_{0}(\tau, .)\right\|_{\infty}^{2} \\
& +\frac{1}{\omega} g^{2} \bar{N}(\tau)^{2}+l^{2}\left\|\frac{\tilde{r}(\tau, .)}{\omega}+\tilde{p}(\tau, . . .)\right\|_{L^{2}}^{2} .
\end{aligned}
$$

With use of Proposition 5.4, Lemma 5.1 and the above estimate (5.19) we arrive at

$$
\begin{aligned}
\left\|\mathscr{L}_{\alpha} P(\tau, ., .)\right\|_{L^{2}(\Omega \times V)}^{2} \leqslant & \omega|\Omega|\left(\kappa_{\alpha, \beta} K_{\vartheta, T}\left\|N_{0}(\tau, .)\right\|_{C^{0, \sigma}}+K_{2}\left(N^{*}\right)\right)^{2}+\frac{\omega|\Omega| s^{2}}{v_{\alpha}} K_{\vartheta, T}\left\|N_{0}(\tau, .)\right\|_{C^{0, \sigma}} \\
& +\frac{1}{\omega} g^{2} e^{2 g T} \bar{N}(0)^{2}+c_{6} l^{2} \bar{N}(0)^{2}
\end{aligned}
$$

which is bounded independent of $\varepsilon$. We denote the right-hand side

$$
c_{7}=c_{7}\left(|\Omega|, T, \vartheta, \alpha, \beta, v_{\alpha}, s, N^{*}, \bar{N}(0),\left\|N_{0}(0, .)\right\|_{C^{0, \sigma}}\right) .
$$

Finally, we split $P$ according to

$$
P=\frac{1}{\omega} \int P d v+\tilde{P}
$$

Then $\mathscr{L}_{\alpha} P=\mathscr{L}_{\alpha} \tilde{P}=Z \in\langle 1\rangle^{\perp}$ and $\mathscr{F}_{\alpha} Z=\tilde{P}$. For $\tilde{P}$ we obtain

$$
\|\tilde{P}(\tau, ., .)\|_{L^{2}(\Omega \times V)}^{2}=\left\|\mathscr{F}_{\alpha} Z\right\|_{2}^{2} \leqslant\left\|\mathscr{F}_{\alpha}\right\|^{2}\|Z\|_{2}^{2} \leqslant \frac{c_{7}}{v_{\alpha}^{2}}
$$

Hence $\|\tilde{P}(\tau, . .,)\|_{L^{2}(\Omega \times V)} \leqslant \sqrt{c_{7}} / v_{\alpha}$. Since $\int P d v$ is bounded by $R$ via (5.12) an upper bound for $P$ results.

Finally, we switch to the original time and space variables $(t, x)$ and obtain the assertion of the Theorem.

Remark 5.6 We cannot expect to get the strong approximation property as in Theorem 5.5 for general nonlinear rates $\tilde{g}(N), \tilde{l}(N)$. For most relevant nonlinear cases, the transport system (1.2), (1.3) and the parabolic limit (5.4), will both have a global compact attractor. It is not clear, however, how these attractors are related. If, for example, the attractor of the parabolic limit equation is chaotic, then trajectories are sensitive to perturbations. Each approximation of solutions will fail after a certain time. Even if the diffusion limit has a stable limit-cycle it is not clear that solutions of the transport model and the corresponding parabolic approximation enter the limit cycle with exactly the same phase. To get more insight into the nonlinear case one has to consider upper and lower semi-continuity of the corresponding attractors. 


\section{Various special cases}

\subsection{Perturbations of homogeneous initial data}

In the previous section we have seen that the outer solution is a good approximation to the resting-phase transport equation for homogeneous initial conditions (matching). Here we show that for constant $\alpha$ the solutions of the resting-phase transport equation (1.2), (1.3) for initial data of the form $p(0, x, v)=\varphi(x)+\varepsilon \varphi_{1}(x, v)$ are of order $\varepsilon$ compared to the solution of the corresponding homogeneous problem with $p(0, x, v)=\varphi(x)$. This result holds for general, nonlinear kinetics, as specified in assumption (A1).

Theorem 6.1 Assume (A1)-(A5). Suppose that

(1) $(r, p)$ solves $(1.2,1.3)$ with initial conditions $p(0, x, v)=\varphi(x)+\varepsilon \varphi_{1}(x, v)$ and $r(0, x)=$ $\psi(x)$ with $\int_{V} \varphi_{1}(x, v) d v=0$.

(2) $(a, b)$ solves $(1.2,1.3)$ with initial conditions $a(0, x, v)=\varphi(x)$ and $b(0, x)=\psi(x)$.

Then for each $T$ there is a constant $c_{8}$ such that for all $t \leqslant T$

$$
\|p(t, . . .)-a(t, . . .)\|_{L^{2}\left(\mathbb{R}^{n} \times V\right)}+\|r(t, .)-b(t, .)\|_{\infty} \leqslant \varepsilon c_{8}\left\|\varphi_{1}\right\|_{D(\mathscr{A})} .
$$

Proof We study the difference $y:=p-a, z:=r-b$ and we denote $N(t, x):=\int p(t, x, v) d v+$ $r(t, x)$ and $M(t, x):=\int a(t, x, v) d v+b(t, x)$. Then $(y, z)$ solves

$$
\begin{aligned}
y_{t}+v \cdot \nabla y & =\mathscr{L} y-\alpha y+\frac{\beta}{\omega} z-l(N) p+l(M) a \\
z_{t} & =\alpha \int_{V} y d v-\beta z+g(N) r-g(M) b-l(N) r+l(M) b \\
y(0, x, v) & =\varepsilon \varphi_{1}(x, v) \\
z(0, x) & =0 .
\end{aligned}
$$

Since $N-M=\int(p-a) d v+r-b=\int y d v-z$, we rewrite this system as

$$
\begin{aligned}
y_{t}+v \cdot \nabla y= & \mathscr{L} y-\alpha y+\frac{\beta}{\omega} z-l(N) y-a \frac{l(N)-l(M)}{N-M}\left(\int y d v+z\right) \\
z_{t}= & \alpha \int_{V} y d v-\beta z+g(N) z+b \frac{g(N)-g(M)}{N-M}\left(\int y d v+z\right) \\
& -l(N) z-b \frac{l(N)-l(M)}{N-M}\left(\int y d v+z\right) \\
y(0, x, v)= & \varepsilon \varphi_{1}(x, v) \\
z(0, x)= & 0 .
\end{aligned}
$$

The functions $g$ and $l$ are supposed to be uniformly bounded in $C^{1}$. Hence the above system defines a linear evolution equation for $(y, z)$, where the operator $\mathscr{A}$ is perturbed by a bounded, time and space-dependent, multiplication operator. We know already that the initial value problem is solvable since the solutions $(p, r)$ and $(a, b)$ exist. Hence there is a bounded solution operator $\mathscr{2}(t, x): D(\Phi) \times L^{2}\left(\mathbb{R}^{n}\right) \rightarrow L^{2}\left(\mathbb{R}^{n} \times V\right) \times L^{2}\left(\mathbb{R}^{n}\right)$ such that 
the solution can be written as

$$
\left(\begin{array}{c}
y(t, x, v) \\
z(t, x)
\end{array}\right)=2(t, x)\left(\begin{array}{c}
\varepsilon \varphi_{1}(x, v) \\
0
\end{array}\right)
$$

Then the assertion follows with $c_{8}:=\sup _{t \leqslant T}\|\mathscr{Q}\|_{\mathscr{L}\left(D(\Phi), L^{2}\right)}$.

\subsection{Small proportion of the population in resting phase}

In case of $\alpha \rightarrow 0$ individuals do not stop moving and hence in our model they cannot reproduce. For the relevant operators and parameters we observe that for $\alpha \rightarrow 0$ (we will not specify any norms for convergence of these operators).

$$
\begin{aligned}
\mathscr{L}_{\alpha}=-(\mu+\alpha) I+(\mu+\alpha) \mathscr{T}_{\alpha} & \longrightarrow-\mu I+\mu \mathscr{T} \\
\mathscr{F}_{\alpha} & \longrightarrow \mathscr{F} \\
D_{\alpha, \beta} & \longrightarrow D=-\frac{1}{\omega} \int v \mathscr{F} v d v \\
N_{0} & \longrightarrow \omega p_{0}
\end{aligned}
$$

Then the limit equation (3.14) reduces to

$$
N_{0, \tau}=\nabla D \nabla N_{0}-\tilde{l}\left(N_{0}\right) N_{0}
$$

which is a reaction-diffusion equation for the pure death process with diffusion tensor $D_{0}$ as in the cases studied in Hillen \& Othmer [13].

\subsection{Large proportion of the population in resting phase}

There is a large proportion of the population in the resting phase if $\alpha$ is large and $\beta$ is small. In that case $\alpha /(\alpha+\beta) \approx 1$ and the limit equation (3.14) becomes

$$
N_{0, \tau}=\nabla D_{\alpha, \beta} \nabla N_{0}+\tilde{f}\left(N_{0}\right)
$$

The complementary factor $\beta /(\alpha+\beta)$ is small, which shows that the modified diffusion tensor $D_{\alpha, \beta}$ given by (3.15) is small. To be more specific we consider an example.

Example: $\boldsymbol{V}=\boldsymbol{s} \boldsymbol{S}^{\boldsymbol{n}-1}, \boldsymbol{T}=\mathbf{1} / \boldsymbol{\omega}$ Then $T_{\alpha}=1 / \omega$ and the pseudo-inverse $\mathscr{F}_{\alpha}$ is a multiplication operator by $-(\mu+\alpha)^{-1}$. Then

$$
D_{\alpha, \beta}=\frac{\beta}{\omega(\alpha+\beta)} \int v v d v \frac{1}{\mu+\alpha}=\frac{s^{2}}{(\mu+\alpha) n} \frac{\beta}{\alpha+\beta} I .
$$

The diffusion tensor is isotropic and the limit equation reads

$$
N_{0, \tau}=d_{\alpha} \Delta N_{0}+\tilde{f}\left(N_{0}\right), \quad \text { with } \quad d_{\alpha}=\frac{s^{2}}{(\mu+\alpha) n} \frac{\beta}{\alpha+\beta} .
$$

For $\alpha=0$ this reduces to $d_{0}=\frac{s^{2}}{\mu n}$. Hence increasing $\alpha$ reduces the motility.

Remark 6.2 It is easy to see that birth-death terms of order less or higher than $\varepsilon^{2}$ will not lead to a limit equation like (3.14): 
(1) For perturbations of the form $f(u)=\varepsilon \tilde{f}(u)$ the nonlinearity $f$ would enter into the turning operator $\mathscr{L}_{\alpha}$ in the equation for $\varepsilon^{1}$. This is longer a linear problem and we cannot apply the framework of Fredholm operators as used here.

(2) For higher order perturbations $f(u)=\varepsilon^{3} \tilde{f}(u)$ the nonlinearity would not enter at all and the limiting equation would be linear to second order with a vanishing reaction part.

\subsection{A parabolic model with resting phase}

As an illustration we compare the previous results with a model where particle movement is described by an uncorrelated random walk (Brownian motion). We keep the assumptions of $\S 1$ with the exception of assumption 1 . Instead we assume now that

1. The movement of the population is described by the diffusion equation $u_{t}=d \Delta u, d>0$ Then the parabolic model with resting phase reads

$$
\begin{aligned}
u_{t} & =d \Delta u-\alpha(x) u+\beta r-l(N) u \\
r_{t} & =\alpha(x) u+g(N) r-\beta r-l(N) r \\
N(t, x) & =u(t, x)+r(t, x)
\end{aligned}
$$

Special cases of this model have been studied for invasion speeds [12, 16] and for protein dynamics [5].

We consider the same scaling as above for the outer expansion

$$
\tau=\varepsilon^{2} t, \quad \xi=\varepsilon x, \quad f(u)=\varepsilon^{2} \tilde{f}(u) .
$$

Then the scaled system reads.

$$
\begin{aligned}
& \varepsilon^{2} u_{\tau}=\varepsilon^{2} d \Delta_{\xi} u-\tilde{\alpha} u+\beta r-\varepsilon^{2} \tilde{l}(N) u \\
& \varepsilon^{2} r_{\tau}=\tilde{\alpha} u+\varepsilon^{2} \tilde{g}(N) r-\beta r-\varepsilon^{2} \tilde{l}(N) r .
\end{aligned}
$$

Again, we consider expansions in $\varepsilon$ for $k>2$.

$$
\begin{array}{rlrl}
u(\tau, \xi) & =\sum_{j=0}^{k} \varepsilon^{j} u_{j}(\tau, \xi), & r(\tau, \xi)=\sum_{j=0}^{k} \varepsilon^{j} r_{j}(\tau, \xi), \\
N(\tau, \xi)=\sum_{j=0}^{k} \varepsilon^{j} N_{j}(\tau, \xi), & N_{j}=u_{j}+r_{j} .
\end{array}
$$

A comparison of orders of $\varepsilon$ leads to the following systems:

$$
\begin{aligned}
\varepsilon^{0}: \quad-\tilde{\alpha} u_{0}+\beta r_{0} & =0 \\
\tilde{\alpha} u_{0}-\beta r_{0} & =0 \\
& =0 \\
\varepsilon^{1}: \quad \tilde{\alpha} u_{1}+\beta r_{1} & =0 \\
\tilde{\alpha} u_{1}-\beta r_{1} & =0
\end{aligned}
$$




$$
\begin{array}{ll}
\varepsilon^{2}: & u_{0, \tau}=d \Delta u_{0}-\tilde{l}\left(N_{0}\right) u_{0}-\tilde{\alpha} u_{2}+\beta r_{2} \\
& r_{0, \tau}=\tilde{\alpha} u_{2}-\beta r_{2}+\tilde{g}\left(N_{0}\right) r_{0}-\tilde{l}\left(N_{0}\right) r_{0}
\end{array}
$$

From (6.5), it follows that $r_{0}=\frac{\tilde{\alpha}}{\beta} u_{0}$, hence $N_{0}=\frac{\tilde{\alpha}+\beta}{\beta} u_{0}$. We solve the second equation of (6.7) for $-\tilde{\alpha} u_{2}+\beta r_{2}$ and use this in the first equation of (6.7) to obtain a single equation for $N_{0}$ :

$$
N_{0, \tau}=d \Delta\left(\frac{\beta}{\tilde{\alpha}+\beta} N_{0}\right)+\frac{\alpha}{\alpha+\beta} \tilde{g}\left(N_{0}\right) N_{0}-\tilde{l}\left(N_{0}\right) N_{0},
$$

which can be written in the form:

$$
N_{0, \tau}=\nabla\left(\frac{\beta d}{\tilde{\alpha}+\beta} \nabla N_{0}-\frac{\beta d}{(\tilde{\alpha}+\beta)^{2}} \nabla \tilde{\alpha} N_{0}\right)+\frac{\alpha}{\alpha+\beta} \tilde{g}\left(N_{0}\right) N_{0}-\tilde{l}\left(N_{0}\right) N_{0} .
$$

This equation shows exactly the same scaling in $\alpha$ and $\beta$ as (3.14) and (3.15). The reproduction rate is scaled by the mean proportion of the population which is at rest; the motility is scaled by the mean proportion which is moving; and we obtain a drift toward high values of $\alpha(x)$.

Remark 6.3 The entire scaling analysis also works for death rates which are different in moving and resting stages, $l_{p}$ and $l_{r}$, respectively. It is straightforward to show that the corresponding term in the diffusion limit is the convex combination:

$$
\frac{\tilde{\alpha}}{\tilde{\alpha}+\beta} \tilde{l}_{r}+\frac{\beta}{\tilde{\alpha}+\beta} l_{p}
$$

\section{Conclusion}

In this paper we consider continuum transport models for populations based on individual movement patterns. The model studied here consists of three major processes: (i) a correlated random walk, which is described by a transport equation; (ii) kinetics for the transition from moving to resting, and vice versa; and (iii) kinetics for birth and death. The resulting model (1.2), (1.3) consists of a nonlinear transport equation coupled with an ODE.

As listed in the introduction, many applications of this model are discussed in the literature. These include stream ecosystems, endangered species in fragmented habitats, homeranges, invasion of new species, hunting territories, prey-taxis, bias toward better environments, and protein movement in a cell nucleus.

Here we study (1.2), (1.3) from a mathematical point of view and we apply the theory for the parabolic limit of transport equations which was developed in Hillen \& Othmer [13]. In this paper we show:

- Local and global existence of solutions and positivity using standard semigroup arguments.

- On a macroscopic length scale $\xi=\varepsilon x$ and on a slow time scale $\tau=\varepsilon^{2} t$ the system is well described by the outer solution, which satisfies a reaction-advection-diffusion equation (3.14). 
- The limit equation shows interesting scaling in the relevant parameters. For non constant $\alpha(x)$ there appears a drift-term toward favorable environments. The diffusion coefficient is scaled by the mean fraction of moving particles, whereas the birth term is scaled by the mean fraction of resting particles.

- For homogeneous environments the outer solution approximates the solution of the transport system (1.2), (1.3) to second order in $\varepsilon$.

- The inner solution is driven by the initial conditions and it 'lives' on a fast time scale $t$ and the macroscopic length scale $\xi$. The inner solution converges to a distribution which is homogeneous in $v$, which matches to the initial conditions of the outer solution.

- We consider special cases for perturbed homogeneous initial conditions, for a large fraction of particles in resting or moving states, and we study a diffusion based model which includes resting-moving dynamics.

In further studies we shall apply the theory to the examples mentioned earlier, in particular to bat-territories and to prey-taxis.

Moreover, it would be interesting to see if oriented movement is beneficial for a population, as done by Cosner et al. $[8,2]$ for the parabolic limit model.

We expect that the theory, as used here, can be readily extended to include different death rates for moving and resting compartments, or to include a spatially dependent starting rate $\beta(x)$. It is more of a challenge, however, to include inhomogeneities which show jumps in the quality of the habitat, i.e. jumps in $\alpha(x)$. In (A3) we assumed that $\alpha(x)$ is continuous, which excludes jumps. The functional analysis, which underlies the studies, has to be re-investigated for that case.

\section{References}

[1] Alt, W. (1981) Singular perturbation of differential integral equations describing biased random walks. J. Reine Angew. Math. 322, 15-41.

[2] Belgacem, F. \& Cosner, C. (1995) The effects of dispersal along environmental gradients on the dynamics of populations in heterogeneous environment. Canadian Appl. Math. Quart. 3, 379-397.

[3] Briscoe, B., Lewis, M. \& Parisch, S. (2002) Home range formation in wolves due to scent marking. Bull. Math. Biol. 64, 261-284.

[4] Britton, N. F. (1986) Reaction-Diffusion Equations and Their Applications to Biology. Academic Press.

[5] Carrero, G., McDonald, D., Crawford, E., de Vries, G. \& Hendzel, M. (2003) Using FRAP and mathematical modeling to determine the in vivo kinetics of nuclear proteins. Methods, 29, 14-28.

[6] Carroll, J. \& Lamberson, R. (1993) The owl's odyssey. A continuous model for the dispersal of territorial species. SIAM J. Appl. Math. 53, 205-218.

[7] Chueh, K. N., Conley, C. C. \& Smoller, J. A. (1977) Positively invariant regions for systems of nonlinear diffusion equations. Indiana Univ. Math. J. 26, 373-392.

[8] Cosner, C. \& Lou, Y. (2003) Does movement toward better environments always benefit a population? J. Math. Anal. Appl. 277, 489-503.

[9] Dautray, R. \& Lions, J.-L. (1993) Mathematical Analysis and Numerical Methods for Science and Technology, VI. Springer-Verlag.

[10] Dickinson, R. (2000) A generalized transport model for biased cell migration in an anisotropic environment. J. Math. Biol. 40, 97-135. 
[11] Hadeler, K. P. (1998) Reaction transport systems. In: Capasso, V. and Diekmann, O. (editors), Mathematics Inspired by Biology: CIME Letures 1997, pp. 95-150. Springer-Verlag.

[12] Hadeler, K. P. \& Lewis, M. (2003) Spatial dynamics of the diffusive logistic equation with sedentary compartment. Canad. Appl. Math. Quart. (to appear).

[13] Hillen, T. \& Othmer, H. G. (2000) The diffusion limit of transport equations derived from velocity jump processes. SIAM J. Appl. Math. 61(3), 751-775.

[14] Kareiva, P. \& Odell, G. (1987) Swarms of predators exhibit 'prey-taxis' if individual predators use area-restricted search. Am. Naturalist, 130(2), 233-270.

[15] Ladyžhenskaja, O. A., Solonnikov, V. A. \& Ural’ceva, N. N. (1968) Linear and Quasilinear Equations of Parabolic Type. AMS.

[16] Lewis, M. A. \& Schmitz, G. (1996) Biological invasion of an organism with separate mobile and stationary states: Modeling and analysis. Forma, 11, 1-25.

[17] MüLlER, K. (1954) Investigation on the organic drift in north Swedish streams. Report of the Inst. Fresh Water Reserach, Drottingholm, 34, pp. 133-148.

[18] Murray, J. D. (1989) Mathematical Biology. Springer-Verlag.

[19] Отнmer, H. G., Dunbar, S. R. \& Alt, W. (1988) Models of dispersal in biological systems. J. Math. Biol. 26, 263-298.

[20] Othmer, H. G. \& Hillen, T. (2002) The diffusion limit of transport equations (ii): Chemotaxis equations. SIAM J. Appl. Math. 62(4), 1122-1250.

[21] Pachepsky, E., Lewis, M. \& Lutscher, F. (2003) Persistence, spread and the drift paradox. Theor. Pop. Biol. (submitted)

[22] PaZY, A. (1983) Semigroups of Linear Operators and Applications to Partial Differential Equations. Springer-Verlag.

[23] Stroock, D. W. (1974) Some stochastic processes which arise from a model of the motion of a bacterium. Probab. Theory Rel. Fields, 28, 305-315.

[24] TaYlor, M. E. (1996) Partial Differential Equations III. Springer-Verlag. 